\title{
Neuer Diskus bringt nicht den großen Wurf
}

- Abgenutzte Bandscheiben zu ersetzen, hilft gegen Rückenschmerzen nur wenig mehr als eine konservative Behandlung.

An einer Studie von Christian Hellum et al. vom Universitätskrankenhaus in Oslo hatten sich 173 Patienten beteiligt, die seit mindestens einem Jahr an Rückenschmerzen im Lumbalbereich gelitten hatten und degenerative Veränderungen der Wirbelsäule aufwiesen. Während 87 Probanden drei bis fünf Wochen ambulant mit einer Kombination von Psycho- und Physiotherapie behandelt wurden, legten sich die anderen 86 Teilnehmer auf den Op-Tisch.

Zwei Jahre später verglichen Hellum und sein Team die Werte, welche die beiden Gruppen im Oswestry-Index erzielten einem Fragebogen-gestützten Instrument, mit dem sich körperliche und soziale Einschränkungen durch Rückenbeschwerden auf einer Skala von 0 (keine Einschränkung) bis 100 (bettlägerig) bestimmen lassen.

Das zuvor festgelegte Ziel von 10 Punkten Unterschied wurde verfehlt - der Unter-

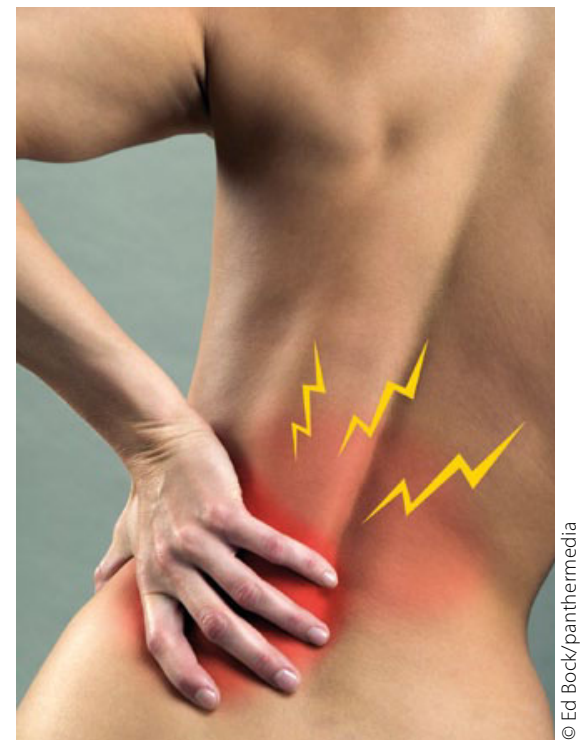

Oswestry-Wert von 21,2 (zuvor 41,8) auf, die konservativ Behandelten einen Score von 30,0 (zuvor 42,8).

Was also tun gegen Kreuzschmerz? Jeremy Fairbank vom Nuffield Orthopaedic Centre der Universität Oxford rät in einem Kommentar zur Studie, in der Frühphase chronischer Rückenschmerzen auf Psycho- und Physiotherapie zu setzen. „Wenn es jedoch zur Operation kommt, spricht nach Hellums Ergebnissen etwas mehr für den Diskusersatz als für die Spondylodese", meint der Wirbelsäulen-Chirurg. Er verweist dabei auf frühere Studien, in denen die Versteifungsoperation mit konservativen Maßnahmen verglichen worden war - und schlechter abgeschnitten hatte als der Diskusersatz im vorliegenden Fall.

Die Lebenszeit-Prävalenz von Rücken$\mathrm{RB}$ schmerzen beträgt bis zu $80 \%$.

schied in der durchschnittlichen Indexverbesserung betrug nur 8,4 Punkte.

Insgesamt schlugen beide Methoden gut an, die Operierten wiesen einen mittleren
Hellum C et al. Surgery with disc prosthesis versus rehabilitation in patients with low back pain and degenerative disc: two year follow-up and randomised study. BMJ 2011; 342: d2786.

\section{Arzt und Anwalt: Man schlägt sich, man verträgt sich!}

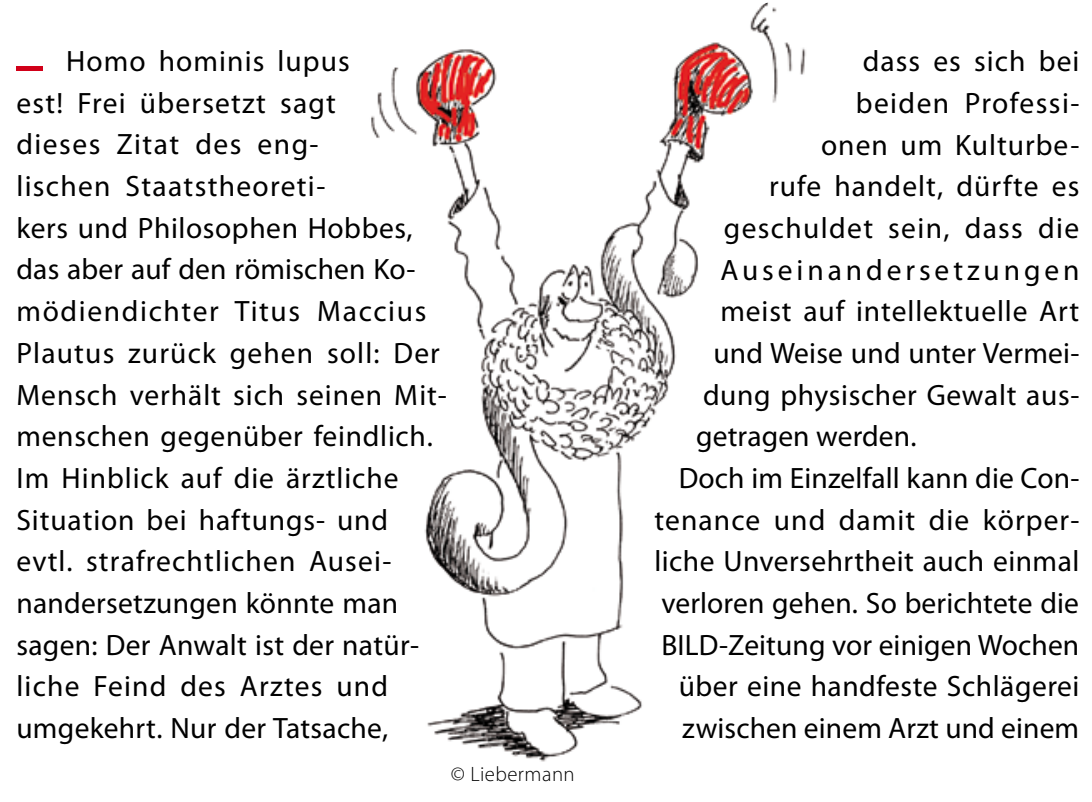

- Homo hominis lupus est! Frei übersetzt sagt dieses Zitat des engStaatstheoretidas aber auf den römischen $\mathrm{Ko}$ mödiendichter Titus Maccius Plautus zurück gehen soll: Der Mensch verhält sich seinen Mitmenschen gegenüber feindlich. Im Hinblick auf die ärztliche Situation bei haftungs- und evtl. strafrechtlichen Auseinandersetzungen könnte man sagen: Der Anwalt ist der natürliche Feind des Arztes und umgekehrt. Nur der Tatsache, Doch im Einzelfall kann die Conenance und damit die körperiche Unversehrtheit auch einmal verloren gehen. So berichtete die BILD-Zeitung vor einigen Wochen über eine handfeste Schlägere zwischen einem Arzt und einem
Rechtsanwalt auf offener Straße. Auslöser war das vermeintlich rechtswidrige Verhalten des anderen im Straßenverkehr. Nachdem entsprechende Verbalinjurien nicht zu einer gegenseitigen Satisfaktion geführt hatten, eskalierte die Sache und beide schlugen heftig aufeinander ein. Physisch schien der Anwalt dem Arzt unterlegen zu sein, sodass er einer sofortigen medizinischen Behandlung bedurfte. So braucht der Anwalt jetzt einen guten Arzt und der Arzt wohl einen guten Anwalt. Und in Anlehnung an den römischen Komödiendichter lautet das "Fabula docet": Wer sich mit einem Arzt prügelt, braucht einen Arzt.

Dr. Peter Stiefelhagen . 